\title{
La résistance des hystériques : Bibliothécaires et avocats dans le champ de la sécurité nationale américaine
}

'Hysterics' resistance: US lawyers and librarians in the field of the professionals of security

\section{Florent Blanc}

\section{(2) OpenEdition Journals}

\section{Édition électronique}

URL : http://journals.openedition.org/conflits/18250

DOI : $10.4000 /$ conflits. 18250

ISSN : $1777-5345$

\section{Éditeur :}

CCLS - Centre d'études sur les conflits lilberté et sécurité, L'Harmattan

\section{Édition imprimée}

Date de publication : 31 décembre 2011

Pagination : 81-102

ISBN : 978-2-296-96617-8

ISSN : 1157-996X

\section{Référence électronique}

Florent Blanc, «La résistance des hystériques : Bibliothécaires et avocats dans le champ de la sécurité nationale américaine », Cultures \& Conflits [En ligne], 84 | Hiver 2011, mis en ligne le 15 mars 2013 consulté le 30 mars 2021. URL : http://journals.openedition.org/conflits/18250 ; DOI : https://doi.org/ 10.4000/conflits. 18250 


\section{La résistance des hystériques 1 : Bibliothécaires et avocats dans le champ de la sécurité nationale américaine}

\section{Florent BLANC}

Florent Blanc est docteur en sciences politiques, diplômé de l'Institut d'Études Politiques de Paris et de Northwestern University. Ses recherches se concentrent sur les études de sécurité, en mêlant analyse des politiques et pratiques de lutte contre la menace terroriste et observation de l'évolution des formes d'action collective en période de crise politique. En poste dans une ONG française, il intervient régulièrement auprès d'universités mais aussi d'organisations de défense des droits de l'homme sur la question des résistances aux pratiques du contreterrorisme. Florent Blanc est chercheur associé à la Chaire Tocqueville d'Études de Sécurité de l'Université de Namur avec laquelle il travaille autour de la notion de désécurisation.

$\ll \int \begin{aligned} & \text { Amérique est en guerre ». Cette phrase apparait au cour même du } \\ & \text { discours politique américain sur la sécurité le } 11 \text { septembre 2001, }\end{aligned}$ et plus encore après le 14 septembre. Clé de voûte du dispositif mis en place dans les jours, les semaines et les mois qui suivent les attentats, c'est une phrase qui ne se discute pas ou alors à mots couverts, même dix ans après. L'idée d'une guerre contre le terrorisme, pour contesté qu'ait pu être le concept dans la littérature des sciences sociales, ne fait l'objet que de peu d'opposition sur la scène politique et dans les cénacles où se discutent ces décisions. Une association comme celle-ci conditionne une vision particulière $\mathrm{du}$ monde, et prédispose celui qui l'accepte à ne pas questionner les mesures qui en découlent. "L'Amérique en guerre » naît, dans les discours officiels, de l'adoption de l'Authorization for Use of Military Force (AUMF) du $14 \mathrm{sep}$ -

1. Ce qualificatif renvoie à une remarque faite par John Ashcroft le 15 septembre 2003 à propos des inquiétudes exprimées par les bibliothécaires au nom de la défense des libertés individuelles. Ses propos exacts étaient les suivants: «les accusations lancées par l'Association des Bibliothécaires Américains contre le gouvernement [à propos de l'accès aux fichiers d'usagers] alimentent une hystérie sans fondements ». Voir à ce propos, Eric Lichtblau, "Ashcroft Mocks Librarians and Others Who Oppose Parts of Counterterrorism Law", New York Times, 16 septembre 2003. Le lecteur comprendra que le titre choisi ici n'est en aucun cas négatif. 
tembre ${ }^{2}$. C'est par cette mesure législative succincte - elle ne représente pas plus d'une page de texte - que le Congrès américain décide d'autoriser le chef de l'exécutif et commandant en chef à employer les forces armées pour lutter contre « les individus et les groupes responsables d'avoir conçu, autorisé, planifié, commis et aidé à commettre les attentats du 11 septembre ». Si beaucoup se sont penchés sur le fait de savoir si cette mesure constituait ou non une déclaration de guerre en bonne et due forme, et si une guerre au sens classique du terme pouvait réellement être déclarée contre un ennemi autre qu'un État, l'intérêt de la mesure n'est pas exactement là. Il se situe ailleurs, dans l'emploi qui va être fait de cette législation exceptionnelle pour légitimer un véritable arsenal de mesures d'exception. Devenue l'élément central du discours sur la sécurité, l'AUMF permet à l'exécutif de décider seul de l'usage approprié de la force militaire dans le cadre de la lutte contre le terrorisme. Les références à l'AUMF, que l'on retrouve dans un ensemble de mesures allant de la décision d'intervenir en Irak à l'élimination d'Oussama Ben Laden, en passant par le scandale des écoutes de la National Security Agency (NSA), démontrent la centralité du lien fait le 14 septembre entre lutte contre le terrorisme et mobilisation des forces armées ${ }^{3}$. Pilier fondateur des efforts de légitimation des mesures d'exception adoptées par la suite, l'AUMF constitue la poupée russe initiale, celle autour de laquelle viendront se greffer d'autres mesures d'exception suivantes. 4

2. "To authorize the use of United States Armed Forces against those responsible for the recent attacks launched against the United States", Senate Joint Resolution, Public Law 107-40, 18 octobre 2001.

3. La référence à l'AUMF est ainsi évoquée pour légitimer l'autorité du Président, en tant que commandant en chef des forces armées, à placer en détention illimitée les personnes désignées comme ennemis combattants (voir les arguments du gouvernement lors des décisions de la Cour Suprême Hamdi v. Rumsfeld en 2004 et Padilla v. Rumsfeld en 2004). L'autorisation $\mathrm{du}$ Congrès d'employer la force militaire pour lutter contre la menace terroriste va être invoquée également pour légitimer l'intervention américaine en Irak comme le texte de la House Joint Résolution to Authorize the Use of the United States Armed Force Against Iraq (H.J.Res. 114 of 2002). Les paragraphes introductifs de ces décisions législatives constituent en effet la liste des arguments légaux de légitimation de la loi. Parmi ceux-ci figurent ainsi de manière proéminente l'AUMF du 14 septembre 2001 qui autorise le Président à employer la force armée pour lutter contre la menace terroriste « responsable des attentats du 11 septembre ». Une AUMF sert donc à légitimer la suivante. Le texte est consultable à l'adresse suivante:http://www.gpo.gov/fdsys/pkg/BILLS-107hjres $114 \mathrm{enr} / \mathrm{pdf} /$ BILLS-107hjres114enr.pdf. Enfin, l'AUMF du 14 septembre 2001 est invoquée pour justifier les interceptions électroniques de la NSA pratiquées sans mandat. Dans un white paper sur le sujet, le Département de la Justice invoque de manière plus qu'explicite l'AUMF du 14 septembre 2001 pour justifier l'autorisation donnée par le Président à la NSA pour pratiquer ces interceptions. Voir à ce propos, "Legal Authorities Supporting the Activities of the National Security Agency Described by the President", US Department of Justice, 19 janvier 2006. Enfin, l'AUMF du 14 septembre 2001 sera de nouveau évoquée à la suite de l'opération militaire menée par les forces américaines en territoire pakistanais, le 1er mai 2011. Cette opération, dans laquelle Oussama Ben Laden a trouvé la mort, a fait l'objet de questionnements quant à sa légalité et ses justifications, notamment dans les milieux juridiques et politiques. L'effort de justification légale de cette intervention vient de Harold Koh, conseiller juridique de la Maison Blanche et ancien dean de la Yale Law School. Son argumentaire, reprit entièrement sur le site OpinioJuris, invoque la légalité de cette opération au nom de l'autorité conférée au Président par l'AUMF du 14 septembre 2001. Le texte est disponible à l'adresse suivante : http://opiniojuris.org/2011/05/19/the-lawfulness-of-the-us-operation-againstosama-bin-laden/ 
Ainsi, malgré les efforts de résistance déployés par les membres des commissions d'examen des lois du Congrès américain, le discours de l'administration Bush va, en s'appuyant sur cette mesure initiale, parvenir à légitimer l'acceptation du Patriot Act. À peine quelques semaines plus tard, aidé en cela par les juristes de l'Office of Legal Counsel (OLC) ${ }^{5}$, l'administration Bush jette les bases d'une véritable justice militaire d'exception qui retire de fait aux prisonniers toute protection juridique alors que le camp où ils sont détenus est soustrait de toute juridiction 6 .

Composante essentielle de l'effort continu de légitimation de ces pouvoirs d'exception, un ensemble de discours et de pratiques visant à dissuader toute opposition politique est mis en place insidieusement. Alors que les membres des commissions d'examen des lois du Congrès avaient été accusés de ralentir l'effort de protection contre la menace terroriste en refusant de valider sans examen la proposition de loi du gouvernement, d'autres, comme les avocats des détenus, se verront accusés publiquement par Cully Stimson, DeputyAssistant Secretary of Defense, en 2007, de soutenir les terroristes en voulant les défendre ${ }^{7}$.

Face à la mise en œuvre d'une stratégie, non pas de dépolitisation, mais bien de captation de pouvoirs au nom de la sécurité nationale, il nous semble intéressant aujourd'hui de se pencher sur les mécanismes mais aussi sur les contre-stratégies déployées par un certain nombre d'acteurs pour s'opposer à l'administration Bush durant cette crise de sécurité. Le terme d'opposition est ici réducteur puisque, comme nous le verrons, il s'agit plutôt d'un questionnement sans répit qui vise à forcer l'administration à justifier et légitimer les pouvoirs d'exception que lui a consentis le Congrès.

En choisissant de prendre de la distance par rapport aux contributions théoriques sur le sujet, celle-ci fait le choix de permettre une compréhension sociologique fine de la période post-11 septembre pour en illustrer les mécanismes politiques. Plutôt que d'aborder les littératures relatives à l'exception et à la sécurisation, c'est à celle qui concerne la notion de résistance que nous

4. Uniting and Strengthening America by Providing Appropriate Tools Required to Intercept and Obstruct Terrorism (USA PATRIOT ACT) Act of 2001, Public Law 107-56, 107 th Congress.

5. L'OLC est intégré au sein du département de la Justice et assiste le secrétaire à la Justice dans son rôle de conseiller juridique de l'exécutif. Composé de membres nommés politiquement, il rend des avis consultatifs.

6. Voir en particulier les memoranda les plus importants : Philbin Patrick, Memorandum for Alberto Gonzales, Counsel to the President: Legality of the Use of Military Commissions to Try Terrorists, mais aussi Yoo John, Memorandum to Jim Haynes II: Application of Treaties and Laws to Al Qaeda and Taliban Detainees, 9 janvier 2002. Et enfin, celui qui marque le début de la réflexion sur l'emploi de la torture : Jay Bybee (OLC) pour Jim Haynes II (DoD) daté du 26 février 2002, Potential Legal Constraints Applicable to Interrogations of Persons Captured by US Armed Forces in Afghanistan.

7. Voir l'éditorial du Washington Post à ce propos: "Unveiled Threats", Washington Post, 12 janvier 2007 
avons souhaité consacrer notre attention en mobilisant les apports historiques et sociologiques qui permettent de saisir l'origine des valeurs et des habitus mobilisés en période de crise. Si la sécurisation n'est pas le résultat d'un speech act, la résistance doit également se comprendre comme un effort patient de déconstruction des discours sur la crise de sécurité et plus encore un effort stratégique en vue de limiter les possibilités de discours du pouvoir sur l'exception nécessaire, pour progressivement venir encadrer, de nouveau, la pratique du pouvoir par les règles du droit.

L'analyse des discours et des pratiques de résistance et de contre-résistance, au cœur de cette contribution, a pour but de donner à voir une analyse empirique sociohistorique qui permette de saisir les jeux d'acteurs au sein du champ des professionnels de la sécurité.

En se concentrant sur deux mesures particulières de sécurité que sont le Patriot Act et la politique de détention mise en œuvre dans le cadre de la guerre contre le terrorisme, cette contribution vise à analyser les producteurs de discours de résistance et d'opposition aux arguments du gouvernement et de ses soutiens. Nous avons choisi deux groupes distincts, tant par leur composition que par leurs buts, leurs fonctions, la sociologie de leurs membres ou encore leur relation au pouvoir. Précurseurs et tête de réseau entre 2001 et 2009, l'American Library Association (ALA) et la coalition d'avocats formée autour du Center for Constitutional Rights - qui a assuré la coordination des avocats américains volontaires pour assurer la défense des détenus de Guantanamo - vont permettre d'analyser les répertoires d'action mis en œuvre dans le but d'empêcher l'administration Bush de commettre des abus de pouvoir. Membres d'une société civile organisée et porteurs de valeurs politiques qu'ils veulent défendre, c'est par la mobilisation des approches sociologiques sur les mouvements sociaux et la participation politique que nous aborderons l'analyse de leurs pratiques de résistance.

\section{Résister comme un phare dans le brouillard : le « texte caché » (bidden transcript) de la sécurité}

Sans tomber dans le manichéisme le plus basique, force est de constater, en empruntant une analyse en terme de champs, que l'enjeu de la joute discursive est bien celui d'imposer un discours qui valide un certain nombre de mesures politiques. Une fois la domination d'un discours établie et acceptée, c'est un effort de validation et de légitimation qui est produit pour garder ce contrôle sur le discours de la menace et sur la manière d'y répondre.

La métaphore contenue dans le titre permet d'introduire l'idée selon laquelle la résistance, dans ce cas précis, est l'œuvre d'acteurs capables, grâce à leur socialisation professionnelle, de voir au travers du «brouillard ». Ce brouillard est celui jeté par les juristes de l'administration Bush mais aussi la 
rhétorique de l'unité nationale, qui tend à masquer les actions du gouvernement, à dissuader les dissidences en les traitant comme des déviances politiques, mais aussi à construire une légalité alternative qui prévient la nécessité de transparence démocratique. Dans une logique proche de celle de James C. Scott ${ }^{8}$, l'imposition d'un « texte public » (public transcript), composé des discours officiels sur la sécurité, s'accompagne d'un effort pour réprimer l'énonciation sur la scène publique du contenu du « texte caché », formé des discours de contestation émis par les acteurs dont la voix, au sein du champ, ne peut se faire entendre.

Au lendemain des attaques terroristes du 11 septembre 2001, des voix s'élèvent, en privé mais aussi très rapidement au sein de l'espace public, pour mettre en garde contre les tentations d'une réaction excessive. Ces voix, à l'instar du conducteur du métro de Bourdieu ${ }^{9}$, sont celles de citoyens qui, malgré l'émotion, continuent de réfléchir et de se penser en société. Ces voix seront aussi celles d'hommes politiques, de commentateurs politiques, d'avocats, d'associations de défense des libertés. Ces profils révèlent une certaine configuration de capitaux sociaux qui donne à leur parole un poids particulier, et qui établit leur autorité sur la question de la sécurité mais aussi sur celle du droit. Plutôt que de tenter l'exercice du catalogage de « ceux qui parlent », il nous a semblé plus pertinent de nous attacher à suivre deux groupes d'acteurs que sont d'une part les bibliothécaires américains, fédérés au sein de l'ALA, et de l'autre une coalition de juristes et d'avocats, dont la coordination sera assurée par le Center for Constitutional Rights.

Si l'Administration et ses soutiens, en tant que locuteurs du discours dominant sur la sécurité, écrivent le public transcript de la sécurité, cette contribution doit s'attacher à mettre en lumière le hidden transcript. Dans une optique scottienne, il existe un autre transcript - ou discours sur la réalité qui représente la vision de la sécurité telle qu'elle est perçue, ressentie, expérimentée, par ceux dont les capitaux, dans le contexte post-2001, ne permettent pas d'investir d'emblée le champ des professionnels de la sécurité. Malgré l'expertise, ou la cause qu'ils défendent, leur parole sera contestée, comme nous l'avons partiellement expliqué plus haut, par les tenants du discours public ou officiel sur la sécurité. C'est donc par l'emploi de techniques et de stratégies que nous détaillerons plus loin que ces acteurs d'un discours de résistance vont prendre d'assaut les frontières du champ et faire peu à peu valoir leur expertise, pour que leur discours se confronte directement à celui des cercles du pouvoir. C'est à ces discours alternatifs que les bibliothécaires et les avocats des détenus de Guantanamo vont contribuer par des arguments qui leur sont propres.

8. Scott J., Domination and the Arts of Resistance: Hidden Transcripts, New Haven and London, Yale University Press, 1990.

9. Bourdieu P., Wacquant L., Réponses : Pour une anthropologie réflexive, Paris, Seuil, 1992. 
Cependant, pour comprendre la nature des acteurs qui se mobilisent à partir de l'automne 2001 contre l'arsenal de mesures de sécurité de l'administration Bush et le risque de déni du droit qu'elles laissent présager, il est important de suspendre brièvement notre examen de la période post-2001 pour fournir des éléments historiques qui permettront au lecteur de mieux saisir la particularité des acteurs de la résistance dont nous parlons ici. En effet, alors que Giorgio Agamben évoque, par le biais d'une citation de Carl Friedrich, l'idée selon laquelle « seule la détermination propre du peuple » permet de s'assurer que les pouvoirs d'urgence sont utilisés dans le seul but de la défense de la Constitution ${ }^{10}$, la question n'en demeure pas moins celle de l'identification de ce peuple. Puisque les observations montrent l'absence de tout mouvement populaire d'opposition à l'agenda sécuritaire américain dans la période de l'après-2001, il convient de s'interroger, par une approche sociohistorique, sur le fait que ce sont des avocats et des juristes réunis au sein d'organisations professionnelles qui vont s'opposer au gouvernement, au nom de la défense des droits et des libertés civiles.

Sans retracer l'histoire complète des relations sociales américaines, il convient d'identifier le processus par lequel les groupes s'opposant aux politiques de sécurité du gouvernement vont adapter leur discours et leur structuration pour lutter plus efficacement et ainsi se donner la chance de faire valoir leur discours sur la scène politique.

Ce processus répond à la nécessité d'éviter la répression du pouvoir tout en se donnant les moyens de parler le même langage que lui. Cette évolution historique consiste à se défaire des attributs du radicalisme politique pour adopter le costume, tant physique que social, des agents du gouvernement et du champ du pouvoir.

C'est en faisant appel au travail d'archives de sociologues comme Michael Rogin ${ }^{11}$, Gary T. Marx ${ }^{12}$ ou Clark McPhail ${ }^{13}$ que l'on peut commencer à retracer l'histoire des stratégies du pouvoir en place pour réprimer les tentatives de revendication par des groupes minoritaires. L'histoire de cette lutte pour la préservation de la structure du pouvoir politique ne peut cependant

10. Friedrich C., Constitutional Government and Democracy, Boston, Ginn, 1950, p. 58, cité dans Agamben G., State of Exception, Chicago, The University of Chicago Press, 2005, p. 8 (nous traduisons).

11. Voir en particulier, Rogin M., Ronald Reagan, the Movie, Berkeley, University of California Press, 1988 ; The Intellectuals and Mccarthy: The Radical Specter, Cambridge, MA, M.I.T. Press, 1967.

12. Marx G.T., Undercover: Police Surveillance in America, Berkeley, University of California Press, 1988.

13. Clark McPhail C., Schweingruber D., McCarthy J., "Policing Protest in the United States: 1960-1995", in Della-Porta D. et Reiter H. (eds), Policing Protest: The Control of Mass Demonstrations in Western Democracies, Minneapolis, University of Minnesota Press, 1998 ; Gilham P.F., Marx G.T., "Complexity and Irony in Policing and Protesting”, Social Justice, $27, n^{\circ} 2,2000$. 
être détachée de celle des mesures de surveillance et de contrôle mises en place depuis les heures sombres des red squads des années vingt, unités de police spécialisées chargées de démasquer les radicaux pour procéder à leur déportation, jusqu'à la surveillance des opposants à la guerre en Irak depuis $2002{ }^{14}$. $\mathrm{Au}$ cours de cette période, ces mesures de contrôle ont fait l'objet d'une contestation auprès des cours de justice américaines qui a notamment permis de préciser, au cours d'une série de décisions prises dans les années cinquante et soixante, la portée du premier amendement de la Constitution ${ }^{15}$. À cette même époque, un ensemble de décisions de la Cour Suprême, regroupées sous la forme d'une doctrine, dénonce sous forme juridique la pratique du pouvoir qui consiste à mettre en place des mesures qui ont pour conséquence directe de dissuader les citoyens d'émettre des opinions politiques contraires au discours dominant 16. Plus proche dans le temps, l'arrêt Handschu de 198317 mais aussi la révélation de la reformation d'unités de police chargées de constituer des dossiers sur les citoyens et les groupes qui protestent contre les mesures de sécurité ${ }^{18}$, sont venus rappeler que les tactiques répressives des forces de police continuent d'évoluer en s'adaptant aux restrictions successives dont elles font l'objet, notamment les Levi Guidelines qui avaient permis d'encadrer de manière stricte les prérogatives du FBI en $1976{ }^{19}$.

Ces techniques visant à empêcher l'expression politique des groupes minoritaires qui contestent les politiques gouvernementales, et a fortiori quand il est question de sécurité nationale, vont conduire les organisations qui mènent et coordonnent ces protestations à évoluer. Ainsi, sous la pression croisée de la nécessité de protester par tous les moyens sans risquer d'être réprimé, mais aussi de financer et d'obtenir le soutien le plus large possible, les organisations représentant les mouvements sociaux font le choix, dans les années soixante, de se professionnaliser 20. Cette évolution, expliquent McCarthy et Zald, répond à la réalisation suivante : pour mener une lutte

14. Donner F., Protectors of Privileges: Red Squads and Police Repression in Urban America, Berkeley, University of California Press, 1990.

15. Les landmark cases suivants méritent l'attention du lecteur : Yates v. United States (1957) et Brandeburg v. Ohio (1969) pour le droit d'expression ; Brown v. Lonisiana (1966) et Adderley v. Florida (1966) pour le droit d'assemblée ; et enfin le droit d'association par NAACP v. Alabama (1958).

16. Chertok M. et Marcus S., "Chilling Political Expression by Use of Police Intelligence Files: Anderson V. Sills," Harvard Civil Rights - Civil Liberties Review 5, n¹, 1970, pp. 71-88.

17. Dans sa décision Handshu v. Special Services Division, la Second Circuit Court de New York met fin aux activités de la Special Services Division intégrée au sein du Département de la police de New York. Cette unité était chargée de constituer des dossiers sur les individus participant à ou organisant des manifestations politiques publiques dans les rues de la ville.

18. TALON et CATIC sont deux bases de données, constituées respectivement par le département fédéral de la Défense et l'Attorney général de Californie. Elles recueillent les informations concernant les menaces de troubles à l'ordre public selon l'idée que ces manifestations publiques pourraient servir de couverture à des actes terroristes.

19. Voir à ce propos Berman E., Domestic Intelligence: New Powers, New Risks, New York, Brennan Center for Justice, NYU Law School, 2011.

20. Voir à ce propos les travaux de John McCarthy et Mayer Zald : The Trend in Social Movements in America: Professionalization and Resource Mobilization, Ann Arbor, Center for Research on Social Organization, University of Michigan, 1977. 
sociale efficace et sur la durée, il est nécessaire pour une organisation de pouvoir bénéficier de personnel à temps complet, et non pas de bénévoles dont la mobilisation ne peut se dérouler qu'en dehors des heures de travail salarié. Par ailleurs, la question de l'efficacité impose le recrutement de spécialistes, recrutés sur un ensemble de compétences techniques. À l'identification de ces besoins correspond un besoin de financement qui dépasse les seules ressources disponibles via la collecte de cotisations versées par les membres. Mais afin de pouvoir présenter des dossiers de financement auprès des grandes fondations philanthropiques qui se développent dans les années soixante, une déradicalisation du discours s'impose au bénéfice d'une rhétorique qui privilégie un changement social incrémental ne remettant pas en cause de manière frontale et directe la structure du pouvoir et de la société qui a permis aux fondateurs de ces institutions d'amasser des fortunes considérables.

La professionnalisation des mouvements sociaux répond également à un besoin que nous avons identifié plus haut : celui de parler le même langage que le pouvoir pour être en mesure d'en contester l'exercice. Bonnie Honig utilise l'idée de «technicalité » ${ }^{21}$ pour expliciter la sélection des acteurs en mesure de remettre en cause les mesures de sécurité adoptées par le gouvernement, et ainsi donner à voir la sociologie des agents capables d'investir le champ de la sécurité depuis ses marges. Cette technicalité, c'est celle du droit administratif et constitutionnel d'abord, qui impose une sélection par la compétence et donc la formation. Mais, chose qu'omet Honig, c'est celle aussi des codes sociaux qui régissent les recours du droit, ces savoir-être qui conditionnent la façon dont un discours sur le droit peut être tenu et surtout entendu. Ainsi donc, la particularité de l'objet contesté et résisté impose une analyse des personnels qui vont être mobilisés pour élaborer un argumentaire d'opposition, dans le cas qui nous intéresse ici, au Patriot Act et aux politiques et pratiques de détention.

\section{Bibliothécaires et crises de sécurité : la construction sociale du devoir de protéger les libertés intellectuelles}

La mobilisation de professionnels répond à l'évolution des répertoires d'action décrite par Tarrow et Tilly 22 dans le contexte particulier des crises de sécurité nationale. Leur position, mais également leur socialisation, leur permet d'agir quand d'autres ne disposent pas des outils et de la mémoire de résistance pour déjouer les jeux de langage du gouvernement sur la sécurité nationale.

21. Honig B., "Bound by Law, Administrative Discretion and the Politics of Technicalities: Lessons from Louis F. Post and the First Red Scare", in Austin S., et Umphrey M. (eds), The Limits of Law, Palo Alto, Stanford University Press, 2005, pp. 209-245.

22. Tarrow S., Tilly C., Contentious Politics, New York, Paradigm Publishers, 2006. 
Aux États-Unis, les intérêts des bibliothécaires sont défendus par une organisation professionnelle, l'ALA, fondée en 1876. L'histoire de cette organisation se lit au travers des crises de sécurité rencontrées par les États-Unis depuis la fin du XIXe siècle. C'est à l'occasion du premier conflit mondial que l'ALA commence à penser son rôle en période de crise, entre source de divertissement pour les soldats, organisation de propagande et défenseur du droit de se cultiver librement. Ce dilemme quant à la position à adopter en période de crise face à un gouvernement qui souhaiterait instrumentaliser les bibliothèques publiques pour servir l'effort de guerre et unir le pays se répète à l'occasion de la chasse aux radicaux des années vingt, de la montée du fascisme et du nazisme en Europe dans les années trente, du second conflit mondial, de la guerre froide et du maccarthysme et enfin au début des années quatre-vingt, quand le FBI se met en chasse des agents soviétiques susceptibles d'utiliser les ressources des bibliothèques pour se renseigner sur les programmes nucléaires américains. Chaque épisode de crise est donc l'occasion privilégiée pour l'ALA d'un questionnement sur le degré de participation à l'effort de guerre ou de lutte contre un ennemi intérieur. L'attitude choisie par l'organisation évolue au gré des expériences participant de l'apparition d'un raisonnement critique en son sein sur les valeurs éthiques à défendre, ainsi que sur la fonction des bibliothèques et des bibliothécaires dans la vie publique et politique américaine. Lors de la Première Guerre mondiale, le choix avait été fait de chercher par tous les moyens à participer à l'effort de guerre afin de démontrer l'importance des bibliothèques et le rôle qu'elles pouvaient jouer en tant que soutien politique - tant à la propagande étatique qu'à la politique de censure destinée à priver l'ennemi intérieur de l'accès à certaines informations stratégiques - et à empêcher l'accès du public à une littérature pro-allemande et « antipatriote». L'ALA se positionne alors comme facteur de lien social avec les soldats déployés en Europe en organisant des collectes et des envois de livres et de revues. Avant même que le conflit ne prenne fin, un courant émerge qui défend une opposition formelle à toute forme de censure quant aux ouvrages mis à disposition des usagers.

L'accession au pouvoir du parti national socialiste en Allemagne en 1933 accélère cette réflexion sur l'éthique, alors que les autodafés du parti nazi multiplient les prises de positions publiques de l'ALA contre la censure sous toutes ses formes, au nom de la défense des libertés intellectuelles. Ces tendances libérales, qui placent l'ALA au cœur d'une réflexion de fond sur la place des libertés fondamentales dans la politique culturelle et sociale américaine, subissent un contrecoup lors de l'entrée en guerre des États-Unis, puisque les instances dirigeantes décident, une fois encore, de servir les buts de la propagande gouvernementale. La pratique des purges se poursuivra avec vigueur à l'époque du maccarthysme quand l'ALA, sous la pression du gouvernement, demandera à ses membres de retirer de leurs étagères les livres 
considérés comme « communistes ou socialistes ». C'est au sortir de cette crise que l'organisation adopte une position forte sur la défense des libertés intellectuelles qui lui permet, dans les années quatre-vingt, de résister aux demandes de coopération émanant du FBI qui visaient à transformer les agents de bibliothèques en adjoints des services de renseignement chargés de dénoncer les usagers ayant des lectures «suspectes ». La position de l'ALA devient alors claire et stricte : rapporter aux forces de police quelconque information concernant les pratiques des usagers des bibliothèques constitue une violation du droit à la vie privée qui pourrait conduire à une limitation des pratiques de recherche intellectuelle. À la fin des années quatre-vingt, l'organisation adopte une série de décisions qui fixent et pérennisent cette ligne politique : le Code of Ethics (1939) ${ }^{23}$, le Library Bill of Rights (1939) ${ }^{24}$ et enfin la Policy on Confidentiality of Library Records (1971) ${ }^{25}$. Ces décisions illustrent l'importance stratégique des fichiers constitués et détenus par les bibliothèques pour les agences de sécurité de l'État. C'est aussi à cette époque que l'ALA envoie à ses membres les premières consignes visant à détruire systématiquement les fichiers constitués une fois les ouvrages restitués, afin de ne pouvoir être mis en position de fournir ces renseignements aux forces de l'ordre.

\section{Quand l'éthique professionnelle s'oppose aux demandes de sécurité}

Au lendemain des attentats du 11 septembre, les bibliothécaires se trouvent plongés au cœur de la crise de sécurité alors que l'enquête établit en quelques jours que les terroristes se sont servi des ordinateurs mis à disposition du public dans les bibliothèques publiques de Floride pour communiquer. Le refus initial des agents de bibliothèque de fournir au FBI la liste des usagers sans présentation d'un mandat officiel déclenche un scandale médiatique. L'ALA réagit pour rappeler à ses membres les consignes éthiques qui insistent sur le fait que la coopération avec les forces de l'ordre est souhaitable, mais dans le respect du droit et des positions éthiques de l'organisation.

Face aux accusations d'antipatriotisme dont ils sont l'objet, les bibliothécaires voient émerger au cœur de leurs débats internes les grandes divisions qui avaient traversé la profession quant à la position éthique à adopter face aux demandes du gouvernement à participer à la lutte contre une menace. L'enjeu que représente l'accès aux dossiers des bibliothèques est mis en avant par la section 215 du Patriot Act qui permet au FBI, sur présentation d'une National Security Letter, d'obtenir les fichiers constitués par une organisation ou une entreprises tout en interdisant à l'employé qui se voit présenter la lettre de révéler l'existence d'une telle demande aux usagers ou à toute autre personne (gag order ${ }^{26}$ ).

23. Au cœur de l'affirmation des valeurs de cette organisation professionnelle, ce code d'éthique a fait l'objet de plusieurs réflexions et amendements en 1981, 1995 et 2008.

24. Celui-ci sera révisé en 1944, 1948, 1961, 1967, 1980 et 1996. 
Forte de son histoire et de la construction de ses valeurs professionnelles, l'ALA va entrer en résistance. Une résistance simple, efficace et moralement inattaquable. Dès le mois d'octobre 2001, l'ALA rappelle à ses membres la nécessité de ne conserver aucun fichier inutile concernant les activités des usagers. Tout fichier doit être détruit une fois les livres retournés. Les fichiers de connexion internet des postes publics doivent être effacés régulièrement 27. Mais à ces consignes émises par leur organisation professionnelle s'ajoutent des actions de résistance individuelles. Certaines bibliothèques publiques, en partenariat avec l'American Civil Liberties Union (ACLU), décident d'informer et de mettre en garde leurs usagers contre l'impact potentiel du Patriot $A c t$, leur suggérant de demander des précisions à leurs élus fédéraux ${ }^{28}$. La mesure visée est la section 215 du Patriot Act, qui permet d'obtenir les fichiers contenant des données personnelles sans contrôle d'un juge. Dans différentes villes, les bibliothécaires organisent des actions publiques au cours desquelles des activistes se relaient pour emprunter et rendre une sélection de livres portant sur les questions de terrorisme, d'islamisme et de fabrication d'explosifs. Le but est d'illustrer la nécessité de défendre le droit à la liberté intellectuelle. En résistant de manière symbolique à la mise en œuvre du Patriot Act, ces bibliothécaires entendent contribuer à faire parler de ces mesures de sécurité de manière critique, et ainsi remettre sur l'agenda public les risques qu'elles comportent. En critiquant ainsi ouvertement et médiatiquement les politiques de sécurité, ces défenseurs de la liberté intellectuelle ont su démontrer que critiquer le discours gouvernemental sur la sécurité en période de crise restait un droit démocratique inaliénable.

Ces actions symboliques qui visent à restaurer un débat public sur le contenu du Patriot Act sont complétées par une campagne de lobbying à l'endroit des représentants et sénateurs afin de les inciter à s'informer sur l'impact de cette mesure de sécurité sur le fonctionnement des bibliothèques. Pour éclairer le débat sur la portée réelle de la section 215, les juristes de l’ALA lan-

25. Cette politique de confidentialité sera amendée en 1975 et 1986.

26. Un gag order se traduit littéralement par « injonction de baillonnement ». Il s'agit d'une provision du droit américain qui interdit à un individu de s'exprimer concernant un acte de droit. Dans le cas du Patriot Act, cette injonction a pour but d'empêcher que le détenteur d'un dossier que les forces de sécurité souhaitent consulter ne puisse être informé d'une action de surveillance et d'enquête en cours. Pour plus de détails, voir "Section 505 - Miscellaneous National Security Authorities”, in USA PATRIOT Act, P.L. 107-56, 26 octobre 2001.

27. Dans son document "Privacy Toolkit: Guidelines for Developing a Library Privacy Policy", l'ALA explique que les bibliothèques n'ont pas vocation à conserver inutilement les données à caractère personnel. Pour éviter la potentialité d'une violation du respect de la vie privée des usagers, l'ALA stipule que " chaque bibliothèque doit prendre les mesures nécessaires pour détruire ces données personnelles ou les convertir de manière à les rendre anonymes » (cf. 4 . Data integrity and security). Le document est consultable à l'adresse suivante : http://www.ala.org/offices/oif/iftoolkits/toolkitsprivacy/guidelinesfordevelopingalibrary privacypolicy/guidelinesprivacypolicy).

28. Voir en particulier la campagne d'information et de mobilisation «campaign for reader privacy ", lancée en 2004 dans le but d'inciter les usagers des bibliothèques à s'informer et à demander à leurs représentants élus de faire adopter une réforme du Patriot Act protégeant les fichiers constitués par les bibliothèques : http://www.readerprivacy.org/ 
cent une campagne dès le printemps 2002 visant à collecter des informations quant à la mise en œuvre de la section 215 par les agents du gouvernement pour obtenir l'accès aux fichiers des bibliothèques ${ }^{29}$. Au nom du droit d'accès aux documents publics (Freedom of Information Act), mais également par le biais de questionnaires envoyés à leurs membres, les juristes de l'ALA tentent d'établir l'utilité pour le gouvernement de cette section.

Ce faisant, l'ALA va parvenir à démontrer le peu d'utilisation en est fait par le gouvernement. Forte de cette conclusion, l'ALA renforce sa campagne visant à obtenir l'abrogation de cette section lors du renouvellement du Patriot Act à l'été $2006{ }^{30}$.

Il est important de noter ici que cette section 215 permet au gouvernement d'avoir accès aux fichiers constitués par le secteur privé en particulier. Sans revenir ici sur l'importance du fichage dont les citoyens, en tant que consommateurs et usagers, font l'objet de manière routinière, on ne peut ignorer le fait que cette mesure visait en priorité les fichiers bancaires et surtout ceux contenant les traces que tout utilisateur d'internet laisse derrière lui. Cependant, aucune protestation ni acte d'information n'a jamais émané de la part des principaux intéressés, à savoir les fameux fournisseurs d'accès à Internet (Internet Service Providers, ISP). En effet, ceux-ci sont les gardiens des fichiers de connexion au réseau qui permettent de capturer la trace qu'un individu laisse derrière lui et qui peut indiquer à qui il s'adresse, ce qu'il lit, la nature de ses recherches. Autant de détails qui jouent un rôle crucial dans une enquête de sécurité. Comment expliquer alors l'absence de protestation des ISP alors que l'ALA s'est mobilisée dès le mois d'octobre 2001 ? Tout simplement par la construction sociohistorique de la relation entre cette organisation et le gouvernement en périodes de crise. Cette histoire est la cause du développement de valeurs et d'une éthique professionnelle qui distinguent l'ALA de toute autre organisation. C'est ce facteur, mais aussi le fait que l'ALA s'est adjoint les services de professionnels du droit capables de déchiffrer les lois, qui a fait de l'ALA un acteur majeur de la résistance au Patriot Act.

29. Les résultats de cette enquête - commanditée par l'ALA - menée auprès de plusieurs centaines de bibliothèques sont publiés dans le rapport d'Abby Goodman, "Impact and Analysis of Law Enforcement Activity in Academic and Public Libraries”, Chicago, American Libraries Association, 2005, 62 p.

30. Soutenus par l'ALA, un groupe de représentants et de sénateurs conduit par Russ Feingold dépose un amendement, le Freedom to Read Protection Act, dès 2003, répondant ainsi à la campagne d'information lancée par l'ALA sous le nom de Campaign for Reader Privacy dont le sous-titre posait la question suivante « is someone reading over your shoulder? ». 


\section{Assurer le droit à être défendu des personnes qui ont déjà été condamnées : le Center for Constitutional Rights et la mobilisation des avocats américains}

I seem to thrive on losing [...] Over the years, we [the Center for Constitutional Rights' legal teams] were spectacularly unsuccessful in court with a few exceptions, we lost every case we litigated. [...] [But our efforts] were successful if they inspire others to struggle, to resist injustice together, and to eschew the easier, more "successful" path ${ }^{31}$.

Jules Lobel, vice-président du Center for Constitutional Rights (CCR)

Compagnon de route des plus grandes batailles juridiques qu'a menées le CCR dans les années quatre-vingt contre le gouvernement américain, Lobel décrit l'acte romantique de ce qu'est bien souvent la résistance dans la littérature. Pourtant, quelques lignes plus loin, il ajoute que s'il a perdu ces procès, les idées qu'il soutenait devant le juge se sont imposées quelques années plus tard. C'est ce type de bataille que les avocats qui se fédèrent autour du CCR dès l'automne 2001 vont livrer contre l'administration Bush au sujet de la légalité du traitement des détenus de la base de Guantanamo Bay à Cuba.

Le CCR a été créé en 1966 dans le but d'assurer le soutien logistique et la coordination des avocats impliqués dans la défense des manifestants du mouvement des droits civiques. Rapidement, les avocats qui le dirigent font du CCR un centre d'expertise légale destiné à soutenir les mouvements sociaux qui œuvrent pour le progrès social. Ils mettent donc leurs compétences techniques et leurs capitaux sociaux au service de causes dans le but d'utiliser les cours de justice pour permettre les changements sociétaux. Dans les années soixante-dix et quatre-vingt, ces avocats s'opposeront notamment au gouvernement américain au sujet de la décision d'intervention à La Grenade ${ }^{32}$ ou encore en ce qui concerne l'imposition de sanctions contre le gouvernement nicaraguayen ${ }^{33}$. L'histoire du CCR et la construction des valeurs des avocats qui y travaillent sont donc cruciales pour comprendre ce que peut être une résistance professionnalisée, et surtout saisir le rôle que cette organisation va pouvoir jouer à l'annonce de l'agenda de mesures sécuritaires de l'administration Bush à l'automne 2001.

31. Lobel J., "Introduction: Losers, Fools and Prophets", in Lobel J. (ed), Success without Victory: Lost Legal Battles and the Long Road to Justice in America, New York, New York University Press, 2003, pp. 8-9.

32. Le lecteur pourra consulter le cas Conyers v. Reagan (1983) qui mettait en cause la constitutionnalité de la décision du Président Reagan de lancer une opération militaire à la Grenade. L'argument présenté par les avocats du CCR, au nom d'une coalition de groupes mais aussi d'une dizaine de congressmen, soulevait un point de non-respect du War Powers Act de 1974.

33. Voir le cas Veterans Peace Convoy v. Schultz (1988) dans lequel le CCR a obtenu d'un juge qu'il signifie à l'Exécutif l'anticonstitutionnalité des mesures prises visant à restreindre ou interdire les dons humanitaires au Nicaragua. 
Les interviews que nous avons pu mener entre 2002 et 2010 auprès d'avocats américains constitutionnalistes et criminalistes impliqués dans la défense des détenus de Guantanamo Bay font ressortir le rapport particulier que le CCR entretient avec les attentats du 11 septembre 2001. En effet, l'immeuble dans lequel se trouvent ses bureaux se situait alors à quelques centaines de mètres à peine des tours du World Trade Center. Le personnel qui avait débuté sa journée de travail a donc connu la peur de la destruction et subit le choc émotionnel de beaucoup de New Yorkais. Malgré ce traumatisme, lorsque le CCR est contacté par un avocat représentant des détenus koweïtis quelques semaines plus tard, l'équipe de direction accepte rapidement d'organiser une conférence téléphonique entre cet avocat, le CCR et un avocat de Minneapolis. Cet appel, qui a eu lieu au mois de novembre 2001, alors que l'armée américaine en Afghanistan commence à ne plus savoir que faire des prisonniers capturés sur le champ de bataille, met en scène une situation juridique dont le cadre n'existe pas. La question que pose l'avocat des Koweïtis est simple : comment s'assurer du sort des détenus capturés et surtout comment organiser leur défense ? L'annonce du Military Order du 13 novembre $2001^{34}$, qui confère au département de la Défense la responsabilité de juger ces détenus, qualifiés de "combattants ennemis » (enemy combatants), par le biais de commissions militaires, suscite les craintes des avocats du CCR. Les conventions de Genève ${ }^{35}$, reprises dans les règles de fonctionnement de l'Armée américaine (US Army Field Manual 36), stipulent que le statut des individus capturés sur le champ de bataille doit être déterminé par un tribunal compétent afin de leur garantir un certain nombre de protections légales minimales. Hors, en Afghanistan, à l'automne 2001, aucune détermination par un tribunal compétent n'est effectuée. Le nombre de prisonniers croit alors. Un nombre considérable d'avocats spécialistes du droit américain vont donc devoir être mobilisés. À la suite de cette conférence téléphonique, le CCR lance donc un appel à la mobilisation auprès des avocats qui sont familiers de ses actions.

Ils sont une poignée à répondre dans les premiers jours, un peu plus à peine dans les mois qui suivront. La mobilisation est lancée mais l'appel reste peu entendu. Parmi ceux qui décident de prêter main forte à l'équipe du CCR, les profils se ressemblent : tous ont défendu des causes progressistes et font donc acte d'engagement militant. Mais leur caractéristique commune principale, c'est l'expérience que beaucoup d'entre eux ont acquise en choisissant de

34. Voir le Military Order of November 13, 2001 : Détention, Treatment and Trial of Certain Non-Citizens in the War Against Terrorism, Washington, White House.

35. Voir l'article 5 de la 3e Convention de Genève sur le traitement des prisonniers de guerre de 1949.

36. Le US Army Field Manual on Interrogation, également connu sous son code FM 34-52, est un manuel qui encadre la pratique des interrogatoires de prisonniers capturés par l'armée américaine. Les consignes qu'il contient intègrent les règles des Conventions de Genève sur le traitement des prisonniers de guerre. Cependant, la décision de Dick Cheney d'août 2002 autorisant l'emploi de techniques dites « augmentées » a impliqué une « mise à jour » de ce field manual par le FM 2-22.3 Human Intelligence Collector Operations. 
défendre des détenus placés dans le couloir de la mort. La bataille contre la peine capitale, nous expliquait l'un d'eux, implique pour un avocat de défendre un détenu accusé d'un crime qui lui vaut la colère du public, de la cour et du jury. Apprendre à travailler contre l'opinion publique, défendre un détenu dont juges et jurés souhaitent l'exécution, c'est comprendre comment trouver les arguments du droit qui permettent à deux membres d'un jury de décider qu'un individu, si horrible que puisse être le crime dont il est accusé, dispose de droits inaliénables et qu'il mérite d'être défendu.

Cette socialisation professionnelle permettra aux avocats qui rejoignent la coalition du CCR de saisir très rapidement que les clients qu'ils sont disposés à défendre gratuitement seront perçus par l'opinion publique comme « le pire du pire », selon les mots du secrétaire à la Défense, Donald Rumsfeld en 2002.

Lors de différents entretiens avec ces avocats, une réponse similaire revenait quant à la raison de leur engagement dans cette bataille judiciaire. "Qui d'autre aurait pu le faire ? " « Nous », répondaient-ils. Qui d'autre qu'un avocat rompu aux fonctionnements des cours de justice, capable de comprendre et de démêler la pelote de laine juridico-politique que les juristes de l'OLC ont tissée autour de Guantanamo ? Leur socialisation, leur expérience, mais aussi les cercles sociaux au sein desquels ils gravitent font en effet de ces avocats les acteurs les plus capables de résister, par l'utilisation du droit, à son détournement au motif de la sécurité nationale ${ }^{37}$.

\section{Vaincre l'exception par le droit, combattre le silence par la plume}

S'il pourrait sembler aisé de porter un regard sur dix années de procédures juridiques pour tenter de contraindre le pouvoir politique américain à simplement appliquer le droit national, militaire et international en ce qui concerne le traitement des détenus, cette facilité n'est que de façade puisque l'examen, même succinct, rélève en réalité d'un travail de Sisyphe. Au cours d'un entretien, un avocat qui avait pris en charge le sort de plus d'une dizaine de détenus yéménites nous avouait la chose suivante : «Depuis le tout début, nous savions que nous avions raison. Nous savions dans le plus cher de notre être que le droit américain n'autorisait pas l'administration Bush à traiter des êtres humains ainsi. Nous savions que nous avions raison, mais encore fallait-il que nous puissions le dire devant un juge prêt à nous entendre ${ }^{38}$ ».

La stratégie mise en place semble plutôt simple : il faut trouver un fil et tirer dessus pour dénouer la pelote. Ainsi, chaque cas poursuit le travail

37. Honig B., op. cit.; Handler J., Social Movements and the Legal System, New York, Academic Press, 1978 ; Luban D., "Lawfare and Legal Ethics in Guantanamo", Stanford Law Review 60, n⿳6, 2008 ; Stone G., Perilous Times: Free Speech in Wartime from the Seditious Act to the War on Terror, New York, W.W. Norton, 2004.

38. Entretien personnel, Chicago, 2007 
engagé par le précédent. Aux sécurisations en poupées russes que nous évoquions dans la première partie de cette contribution répond donc une stratégie juridique en imbrication. Chaque bataille juridique va permettre de défendre un autre cas devant une autre cour de justice et ainsi, lentement, déconstruire les bases mêmes sur lesquelles s'appuie le système de justice militaire et de traitement des détenus.

La première bataille pour faire respecter le droit est celle menée par le Comité international de la Croix-Rouge (CICR). Il s'agit de faire accepter à l'administration Bush et aux autorités militaires la visite du camp de détention et l'examen des détenus. Au titre des Conventions de Genève, le CICR dispose du droit de traverser les lignes de fronts et de rendre visite aux détenus pour s'assurer de leur état de santé, mais aussi et surtout du droit de transmettre un message à leur famille. En remettant une carte postale à chaque détenu du camp de Guantanamo sur laquelle rien de compromettant ne peut être écrit, le CICR a permis aux familles qui en avaient les ressources de contacter un avocat. Ce premier contact va permettre de contourner le refus de l'Administration de communiquer publiquement la liste des personnes placées en détention ${ }^{39}$. Cette première ouverture forcée du camp au monde extérieur permet à certains avocats de se mobiliser. Sans nom, aucun recours n'aurait pu être déposé pour s'enquérir du sort réservé à un détenu.

Dès le début de l'année 2002, alors que le transfert des détenus vers Guantanamo débute, le CCR dépose deux recours en habeas corpus au nom de Shafiq Rasul, Asif Iqbal et David Hicks d'un coté ${ }^{40}$, et de Mamdouh Habib de l'autre ${ }^{41}$. Alors que tous deux ont été capturés en Afghanistan, le CCR demande à la cour du district de Washington de statuer quand à l'interdiction dans laquelle se trouvent les deux détenus de se voir notifier les motifs de leur détention, d'être représentés par un avocat et d'avoir le droit à un procès. Comme l'avaient prévu les juristes de l'OLC dans les mémorandums rédigés à l'automne 2001, la cour du district de Washington se récuse à l'été 2002 en se déclarant incompétente en ce qui concerne le territoire de Guantanamo. Les avocats du CCR font appel de la décision devant la cour d'appel du district de Washington, qui confirme la décision précédente en déclarant qu'aucune cour de justice américaine n'exerce de juridiction sur le territoire de la base de Guantanamo Bay. L'administration Bush semble alors avoir gagné. Mais en septembre 2003, le CCR dépose une motion devant la Cour Suprême des

39. Cette liste ne sera publiée officiellement qu'en 2006 lorsqu'un juge contraindra l'administration Bush à se conformer au droit. Cette décision est intervenue après que des organes de presse (le Washington Post, l'agence Reuters et le New York Times) avaient déposé des pétitions au nom du Freedom of Information Act afin d'obtenir cette information du gouvernement.

40. Les pétitions en habeas corpus déposées pour chacun de ces hommes seront regroupées sous l'appellation unique Rasul v. Bush.

41. Son cas, Habib v. Bush, sera finalement l'objet d'une décision de la Cour Suprême de 2004 également. 
États-Unis, qui se saisit du cas Rasul v. Bush en avril 2004. La décision qu'elle rend en juin 2004 établit qu'en dépit de ce que prétend l'Administration, les détenus étrangers placés sous l'autorité du département de la Défense sur le territoire de la base de Guantanamo Bay, disposent d'un droit d'habeas corpus. Qui plus est, la décision de la Cour autorise les détenus à s'attacher les services d'un avocat civil. Il faudra néanmoins plus de douze mois pour que le premier avocat puisse poser le pied sur le sol de la base militaire. Le département de la Défense impose en effet que chaque civil qui approche les détenus soit détenteur d'une accréditation de sécurité, un processus qui peut prendre plus de six mois dans les cas simples. Par ailleurs, chaque avocat doit être accompagné d'un traducteur assermenté, qui lui aussi a fait l'objet d'une procédure d'enquête et d'accréditation au secret.

La Maison Blanche et le département de la Défense s'engagent alors dans une entreprise d'évitement et de contournement de la décision de la Cour Suprême. Plutôt que d'accorder aux détenus le droit de contester devant une cour de justice leur détention, l'Administration et le département de la Défense instaurent un autre système de justice militaire : les Combatants Status Review Tribunals (CSRT) ${ }^{42}$, dont la mission est de déterminer la culpabilité (et donc le motif de détention continue) des détenus. Défendus par des avocats militaires commis d'office, mis en accusation par des procureurs militaires sur la base d'éléments qui ne satisfont pas nécessairement au régime de la preuve, et jugés par des juges militaires, les détenus n’ont en réalité pas l'ombre d'un procès juste et équitable leur permettant de mettre en œuvre leur droit d'habeas corpus. Moins de deux mois après leur mise en place, les CSRT font l'objet d'une injonction d'une cour de justice qui demande leur suspension ${ }^{43}$. Un an plus tard, la Cour Suprême se saisit du cas Hamdan $v$. Rumsfeld ${ }^{44}$, qui pose la question de la constitutionnalité du pouvoir du président à mettre en place un système de justice ad hoc à Guantanamo. La décision dénonce le manque d'autorité du président. En effet, seul le Congrès peut décider de modifier le droit et donc l'administration de la Justice. Par ailleurs, la décision stipule que les Conventions de Genève s'appliquent à tous les détenus. Cette décision marque donc une victoire éclatante pour les adversaires de l'administration Bush et un sérieux coup porté aux discours de légitimation d'une justice d'exception au nom de l'impératif de sécurité nationale.

42. Les CSRTs sont établis par la décision suivante, Deputy Secretary of Défense, Memorandum for the Secretary of the Navy: Order Establishing Combatant Statuts Review Tribunals, Washington, DC, 7 juillet 2004.

43. Voir la décision de la cour de district de Washington DC, Hamdan v. Rumsfeld de 2004 dans laquelle le juge explique qu'en accord avec les convention de Genève un prisonnier doit être traité comme un prisonnier de guerre tant qu'un tribunal compétent n’a pas établi qu'il en est autrement. Cette décision sera cassée par la cour d'appel du circuit du District de Columbia avant que le cas ne soit porté devant la Cour Suprême en 2006.

44. Voir la décision Hamdan v. Rumsfeld, 548 U.S. 557 (2006). 
La présidence et le camp républicain réagissent en faisant voter le Military Commission Act (MCA) ${ }^{45}$, qui donne l'autorité nécessaire à la poursuite des tribunaux militaires pour juger les détenus de Guantanamo. Alors que la justice militaire d'exception reprend son cours avec de nouvelles règles de fonctionnement et une nouvelle autorité, les avocats réunis autour du CCR, de plus en plus nombreux, continuent de se mobiliser pour faire valoir un droit et un seul : celui de pouvoir présenter une requête en habeas corpus devant une cours de justice américaine.

En décembre 2008, utilisant le cas de Lakhdar Boumedienne ${ }^{46}$, ces avocats mettent en cause la constitutionnalité du MCA de 2006 demandant à la Cour Suprême de statuer sur l'un des piliers des mesures de sécurité adoptées dans le domaine du traitement des détenus : le droit pour le pouvoir exécutif de suspendre l'habeas corpus quel que soit le contexte politique. Ce point du droit est central dans l'histoire du contrôle de l'exécutif et donc pour l'équilibre et la séparation des trois pouvoirs. La Cour déclare, dans sa décision, que le MCA de 2006 ne permet pas l'exercice du droit d'habeas corpus et qu'il constitue donc une suspension illégale de ce droit. Plus de sept années d'actions auprès des cours de justice américaines se sont écoulées depuis l'ouverture du camp de Guantanamo et la mise en ouvre des mémorandums de l'OLC. La Cour, dans sa décision, stipule que les cours de justice américaines sont compétentes à juger les cas ayant pour théâtre la base de Guantanamo Bay. Elle stipule aussi, alors qu'elle n'avait pas osé le faire en 2004, que les citoyens étrangers détenus bénéficient de l'entière protection offerte par les Conventions de Genève, rendant illégale la catégorie d'ennemis combattants.

La décision de la Cour Suprême intervient après la victoire de Barack Obama, dont l'une des promesses de campagne avait été d'accélérer la fermeture du camp de détention de Guantanamo Bay. Les effets de contexte sont donc à prendre en compte, même si la Cour Suprême décide en toute indépendance. En effet, depuis la décision Hamdan v. Rumsfeld de 2006, les avocats des détenus avaient cru sentir venir un changement dans l'opinion publique. Leurs techniques s'étaient diversifiées : en dehors des prétoires et des parloirs, plusieurs d'entre eux avaient saisi leur plume pour décrire le quotidien des détenus, informer l'opinion publique, aider à ce que des détenus rendus anonymes par leurs combinaisons orange, redeviennent aux yeux du public des sujets de droit ${ }^{47}$. C'est aussi à cette période qu'éclate le scandale d'Abu Ghraib, qui révèle les tortures dont sont victimes les détenus, tant en Irak qu’à Guantanamo, mais aussi celui des « restitutions extraordinaires » (extraordinary renditions) par lequel des détenus « de haute valeur » sont transférés vers

45. Military Commission Act of 2006, Pub. L. No. 109-366, 120 Stat. 2600.

46. Voir la décision de la Cour Suprême Boumediene v. Bush, 553 U.S. 723 (2008).

47. Voir notamment le blog de Candace Gorman (http://gtmoblog.blogspot.com/), le recueil de poésie de Mark Falkoff, Poems from Guantanamo: The Detainees Speak, Iowa City, University of Iowa Press, 2007 ; ou encore Ratner M., Ray E., Guantanamo: What the World Should Know, White River Junction, Chelsea Green Pub. Co., 2004. 
des prisons secrètes, dans des pays qui pratiquent la torture. Ces éléments de contexte contribuent à dessiner un paysage politique au sein duquel les arguments de l'Administration ne peuvent plus légitimer les violations du droit au nom de la lutte contre une menace terroriste dont l'actualité décroît pour le public.

Au travers de leurs actions, les avocats sont parvenus à contraindre l'administration américaine à faire la démonstration de la légalité des arguments juridiques qui soutiennent l'arsenal de mesures concernant le traitement des détenus de Guantanamo. Il n'est alors plus question de convaincre un public qui réagit aux arguments de la vitesse ou de l'horreur du crime, mais bien de prouver devant les neuf juges de la Cour Suprême des États-Unis que les politiques mises en œuvre respectent bien la Constitution et la jurisprudence des cours.

Chaque procès est l'occasion pour les avocats de réintroduire, dans leurs arguments, un regard critique non pas sur les discours de l'Administration, mais bien sur l'architecture juridique et logique des pouvoirs arrogés. Confrontés à cette résistance, les défenseurs du gouvernement se voient obliger d'argumenter avec les avocats des détenus qu'ils avaient tenté d'affaiblir sur la scène politique. Peu à peu, malgré la lenteur des cours, de cour de district en cour d'appel et de cour d'appel à la Cour Suprême, les juristes du gouvernement doivent céder du terrain, reconnaitre l'interdiction que le droit leur fait de poursuivre l'idée selon laquelle inter arma silent leges. Les cours sont bien obligées de s'aligner : en temps de guerre, la loi n'est désormais plus silencieuse. L'accumulation de ces décisions en faveur du respect du droit, même en période de lutte contre la menace terroriste, forcera même l'Administration à admettre l'intégration de la jurisprudence internationale dans les pratiques de sécurité du gouvernement américain ${ }^{48}$.

\section{Résistances et désécurisation}

En période de crise de sécurité, quand le gouvernement donne l'impulsion politique en usant de l'urgence comme aiguillon, les acteurs de la résistance, contraints à un silence partiel par l'instauration d'un contexte défavorable mais également par le secret dont le gouvernement entoure les mesures de sécurité, répondent par un acte de résistance premier : celui de parler, de restaurer une parole publique. Cette prise de parole pose les données du problème, affirme les positions et permet d'ancrer l'effort de mobilisation des membres, des proches et des soutiens stratégiques. En parlant, en écrivant, en informant, ces acteurs résistent avant tout à l'implantation du discours dominant dans la mémoire collective afin d'introduire des éléments de doute, de

48. Slaughter A-M., "A Global Community of Courts”, Harvard International Law Journal, 1, 44, 2003 ; Kerch K., "The Supreme Court and International Relations Theory," Albany Law Review, 69, 2006. 
pensée critique. La posture des groupes qui résistent à ces discours peut souvent être résumée par ce mot de Joseph Margulies, l'un des premiers avocats à avoir répondu à l'appel du CCR : " nous étions comme des phares dans le brouillard 49 ». Leurs compétences professionnelles, mais plus encore leur histoire particulière avec l'État et les situations de crise, font de ces acteurs les seuls à disposer de la capacité de prévoir l'attitude du pouvoir et de comprendre la manière de concevoir une stratégie visant à limiter les risques d'abus de pouvoir.

Dans les deux cas que nous avons détaillés plus haut, la stratégie d'action a consisté à exposer les risques d'abus de pouvoir, à les documenter et à les mettre en lumière sur la place publique. À ce travail d'information, destiné à informer le public et ainsi mobiliser les soutiens, une deuxième technique vient s'ajouter. Celle-ci consiste à trouver des recours pour changer la loi. Traditionnellement, pour ce faire, plusieurs moyens existent : la faire invalider par le pouvoir législatif, la faire déclarer contraire à la Constitution par une cour de justice, ou en faire cesser la mise en application par le pouvoir exécutif.

La résistance aux mesures de sécurité post-11 septembre 2001 ne fait pas exception. Les groupes qui résistent - et ils sont bien sûr plus nombreux que ceux que nous avons mentionnés ici - vont employer ces trois voies de recours pour forcer l'Administration à renoncer à gouverner au moyen de mesures d'exception.

Les bibliothécaires, par le biais de la campagne d'information et de résistance publique, mais aussi par l'application d'une stratégie de contestation par les cours de justice ${ }^{50}$, parviendront à médiatiser l'idée selon laquelle le Patriot Act nuit à la liberté intellectuelle des citoyens américains. Le capital de sympathie et d'innocence dont bénéficie, dans la culture américaine, le personnage de la bibliothécaire municipale permettra ainsi de réunir un ensemble de parlementaires résolus à soutenir l'adoption d'un amendement qui interdit l'emploi de la section 215 du Patriot Act pour obtenir les fichiers créés par les

49. Cette citation est tirée d'un entretien personnel conduit en 2007 à Chicago.

50. Voir le cas Library Connection v. Gonzales (2005). Le cas émane de la décision de l'ACLU et de l'ALA de porter devant une cour de justice le cas d'une National Security Letter (NSL) reçue en 2004 par le responsable informatique de Library Connection, laquelle lui demandait de fournir la liste des activités attribuées à un ordinateur identifié par son adresse IP précisément entre $16 \mathrm{~h}$ et $16 \mathrm{~h} 45$ le 15 février 2005. L'action en justice avait pour but de contester le gag order qui interdit au récipiendaire d'un tel courrier de le mentionner même à un avocat. La NSL a été remise le 15 février 2005 alors que le Patriot Act et la section 215 étaient sur le point d'être examinés de nouveau par le Congrès. L'argument présenté par l'ACLU et l'ALA était le suivant : le gag order empêche Library Connection de participer au débat public quant à l'impact du Patriot Act sur les bibliothèques publiques. Ce faisant, il constitue une restriction du droit d'expression et d'information. En septembre 2005, un juge décidera de l'anticonstitutionnalité de ce gag order sans que le gouvernement ne se conforme à la décision. Ce n'est qu'en février 2006, soit trois semaines après la ré-autorisation du Patriot Act, que le gouvernement abandonnera la bataille judiciaire. 
bibliothèques. Lors de la phase de préparation des travaux parlementaires sur la reconduite éventuelle de seize mesures du Patriot Act au printemps 2005, les débats montrent l'ampleur du travail accompli par l'ALA et son équipe de juristes et de lobbyistes ${ }^{51}$. Si les représentants du département de la Justice continuent d'insister sur l'importance cruciale de la reconduction de cette section 215 pour lutter contre la menace terroriste, les parlementaires et aussi les experts auditionnés, sont en mesure de demander au gouvernement de faire la preuve de l'utilité de ce pouvoir d'exception et surtout de forcer le gouvernement à légitimer de nouveau cette mesure concernant les bibliothèques, ce qui implique de légitimer le contournement du pouvoir judiciaire, la violation du droit à la vie privée des utilisateurs et surtout de justifier l'impact dissuasif sur l'exercice des libertés intellectuelles.

Si l'idée d'une menace terroriste reste inébranlée, les moyens mis à disposition du gouvernement sont mis en question, obligeant ainsi l'Administration à participer à un débat partisan. Même les arguments relatifs au danger imminent et à l'unité nationale que le président Bush avait mis en avant pour forcer le Congrès à un vote rapide ne fonctionneront pas. Les débats vont durer près de neuf mois et aboutir à la reconduction de la section 215 pour cinq années supplémentaires, tout en introduisant un certain nombre de mécanismes de contrôle ${ }^{52}$. Si la question des moyens de la lutte contre le terrorisme n'a jamais réellement cessé d'être politique, la reconduction du Patriot Act est l'occasion d'un débat contradictoire par lequel le Congrès fait valoir son pouvoir de contrôle sur l'exécutif. En refusant de signer un blanc-seing à l'Administration sur ces questions, les membres du Congrès condamnent l'équipe Bush à argumenter et à légitimer ces mesures d'exception. Agiter une menace invisible ne suffit donc plus à faire taire la représentation populaire et à lui faire accepter des solutions dont seuls les tenants de l'exécutif peuvent prétendre connaitre l'efficacité.

En ce qui concerne la résistance aux politiques de traitement des détenus, c'est en contraignant le pouvoir judiciaire à se saisir de cas de contrôle de constitutionnalité, et en lui rappelant son devoir de contrôler les actes de l'Administration que les avocats des détenus sont parvenus, après plus de sept années de combat, à rétablir l'affirmation de la primauté du droit en période de crise. L'adage inter arma silent leges n'a donc plus cours, ou presque. Avec l'aide de nombreuses organisations de défense des libertés (ACLU, Amnesty International, Human Rights Watch) mais aussi des grands médias américains (Washington Post, New York Times, Reuters), cette coalition de juristes est

51. Voir à ce propos les auditions menées au cours des débats du Senate Committee on the Judiciary qui se sont tenus du 5 au 10 avril 2005.

52. Voir le USA PATRIOT Improvement and Reauthorization Act of 2005 voté le 14 décembre 2005 par le Sénat et ratifié par le Président le 9 mars 2006. Cette loi, qui prolonge de cinq années les provisions qui étaient arrivées à échéance, introduit un contrôle plus strict du Congrès sur l'utilisation des pouvoirs d'exception et contraint l'Exécutif à rendre compte de l'utilisation de ces pouvoirs plus fréquemment. 
parvenue à lever le voile sur les agissements de l'administration Bush derrière les enceintes des camps de détention et d'interrogation qu'elle avait cru pouvoir légitimer au nom de la guerre contre le terrorisme.

$\mathrm{Si}$, à aucun moment, le discours public n'a vu la remise en cause officielle de l'idée d'une guerre contre le terrorisme, il n'en demeure pas moins que les victoires successives des avocats devant la Cour Suprême, mais aussi la campagne du candidat Barack Obama concernant la fermeture du camp de Guantanamo Bay, traduisent la fin de la confiance accordée à l'administration Bush sur la saillance de la menace et la nécessité impérieuse d'autoriser l'utilisation de pouvoirs d'exception. Pas à pas, les avocats des détenus sont donc parvenus à investir avec succès le champ de la sécurité et à imposer le discours du respect du droit, à faire valoir l'autorité, en période de crise, d'une éthique des droits fondamentaux.

Ce faisant, si la démarche peut sembler froide et méthodique, il n'en demeure pas moins que même au début des années 2000, dans une société démocratique comme celle des États-Unis, résister aux discours du gouvernement comporte un risque personnel pour ceux qui osent prendre la parole publiquement. Lors de l'un de nos derniers entretiens, un des avocats des détenus, devenu professeur de droit dans une grande université américaine, nous faisait écouter un des messages enregistrés la veille sur son répondeur. La menace de mort était claire, les insultes explicites. Résister, c'est peut-être savoir prendre avec humour les insultes et les menaces, tout en admirant, du haut d'une tour de verre, derrière votre bureau, les étudiants qui se pressent pour suivre le récit de votre combat contre l'administration Bush et poursuivre peut-être un jour la bataille du droit en période de crise de sécurité. 